\title{
Model Order Reduction of Time-Delay Systems using a Laguerre Expansion Technique
}

\author{
Elizabeth Rita Samuel, Luc Knockaert, Senior Member, IEEE, and Tom Dhaene, Senior Member, IEEE
}

\begin{abstract}
The demands for miniature sized circuits with higher operating speeds have increased the complexity of the circuit, while at high frequencies it is known that effects such as crosstalk, attenuation and delay can have adverse effects on signal integrity. To capture these high speed effects a very large number of system equations is normally required and hence model order reduction techniques are required to make the simulation of the circuits computationally feasible.

This paper proposes a higher order Krylov subspace algorithm for model order reduction of time-delay systems based on a Laguerre expansion technique. The proposed technique consists of three sections i.e., first the delays are approximated using the recursive relation of Laguerre polynomials, then in the second part, the reduced order is estimated for the time-delay system using a delay truncation in the Laguerre domain and in the third part, a higher order Krylov technique using Laguerre expansion is computed for obtaining the reduced order time-delay system. The proposed technique is validated by means of real world numerical examples.
\end{abstract}

Index Terms-Hankel singular values, Krylov matrix, Laguerre polynomials, Model Order Reduction, Time-delay systems.

\section{INTRODUCTION}

$\mathbf{T}$ HE ever increasing quest for high density and high operating speed requires more accurate models in modern electronic design, and this implies that the analysis of interconnects and high-speed circuits has become a critical aspect for studying system reliability, and speed of operation [1], [2]. The gigabit per second range for high-speed links has made signal integrity (SI) analysis and design of interconnections of great importance.

For printed circuit board (PCB) structures, long transmission lines (TLs) are often needed for accurate simulations. Lossy TLs are traditionally modeled by ladder networks with cascaded sections of RLC components [3]. The effects of different packaging components such as bond wires, traces, vias and balls can also be modeled as RLC lumped components. When geometric dimensions become electrically large and the frequency content of signal waveform increases, time delays must be taken into account and, included in the modeling process [4], [5]. A major drawback of this brute force approach is the potentially huge number of system equations leading to high CPU cost in the circuit simulation. To alleviate this, efficient model order reduction (MOR) techniques can be used.

Elizabeth Rita Samuel, Luc Knockaert and Tom Dhaene are with Ghent University - iMinds, Gaston Crommenlaan 8 Bus 201, B-9050 Gent, Belgium,email: \{elizabeth.ritasamuel, luc.knockaert, tom.dhaene\}@ugent.be

This work was supported by the Interuniversity Attraction Poles Programme BESTCOM initiated by the Belgian Science Policy Office and the Research Foundation Flanders (FWO).

Copyright (c) 2013 IEEE. Personal use of this material is permitted. However, permission to use this material for any other purposes must be obtained from the IEEE by sending an email to pubs-permissions@ieee.org.
Most MOR approaches are based on moment matching techniques (MMTs) and in general, MOR methods can be classified as explicit or implicit MMTs. The explicit MMT using a single Padé expansion [6]-[8] is known as asymptotic waveform evaluation (AWE). However, AWE and the underlying Padé approximation induces numerical ill-conditioning, while the approximant may produce unstable poles. Also, AWE does not guarantee passivity. The implicit MMTs based on Krylov-subspace algorithms [5], [9]-[13] use congruence transformation to deal with ill-conditionedness, and can also guarantee the preservation of passivity in the reduced models.

Time-delay systems (TDSs) are systems with aftereffect or dead-time, which belong to the class of functional differential equations (FDEs), as opposed to ordinary differential equations (ODEs) [14]. TDSs in the Laplace domain contain elements with exponential factors $e^{-s \tau}$ where $\tau$ corresponds to the time delays present in the circuit. In [15], the equivalent representation of the TDSs are build as an infinite-dimensional linear problem and is then reduced to a system without delay. In [16], [17], the exponential terms corresponding to the delays are expanded in a Taylor series and subsequently the moments of the system are obtained via Krylov-subspace techniques. This MOR technique guarantees passivity preservation for certain specific cases of RLC networks by means of congruence transformations. But in [16], [17] the original system needs to be augmented by introducing extra state variables, and finally the resulting reduced order model does not preserve the TDS structure of the original system. In the approach of [5], [12], the moments are calculated using extra state variables, but the dimension of the row of the augmented system moments that is used for the reduction of the original mode is same as the number of state variables in the original model. But still the computation effort is excessive, as the moment matrix is calculated with the extra state variables.

These shortcomings were somewhat alleviated in [13] by proposing multiorder Arnoldi. In that technique the moments of the original system are calculated implicitly using a higher order Krylov technique [18], [19] without having to introduce extra state variables and the structure of the reduced model resembles the original.

In this paper we propose a novel technique using a higher order Krylov subspace decomposition with orthonormal Laguerre functions, which do provide good approximations for a large class of time-delay systems [20]. The link with the singular value decomposition (SVD) leads to a simple and stable implementation of the algorithm. The present technique is a generalization and extension of the well-known LaguerreSVD method [21] to TDS.

As a first step towards improving the efficiency, the proposed technique estimates the reduced order using a smart 
approximation of the delay terms after approximating the delays using a recursive relation using Laguerre polynomials. As a second step higher order Laguerre approximation is used to obtain the reduced order TDS.

More precisely, the three steps of the proposed algorithm are:

1) Delay Approximation - The time delay term $e^{-s \tau}$, is approximated using a recursive relation of Laguerre polynomial.

2) Zero-Order Approximation Technique (ZAT) - The time delay term $e^{-s \tau}$, is approximated as $e^{-2 \alpha \tau}$, where $\alpha$ is the Laguerre parameter [20]-[22]. So an approximate linear time-invariant (LTI) system is obtained, whose Hankel singular values (HSVs) are computed using the Matlab command hsvd. From the HSVs an initial guess of the reduced order can be estimated depending on the required (predefined) accuracy.

3) Higher-order Laguerre Approximation Technique (HLAT) - The delays are replaced by their approximations in the Laguerre domain and then the higher order Krylov subspace is computed [23] in order to reduce the original system.

Thereby, the proposed Laguerre expansion technique computes the Krylov subspace more efficiently, and generates an accurate reduced order model.

This paper is organized as follows. Section II gives an overview of classical Laguerre-based MOR. Section III describes the proposed MOR algorithm for TDS and proposes a flowchart with the algorithmic steps of the novel approach. Section IV validates the proposed algorithms by means of real world examples and by comparing the proposed technique with some recent techniques of MOR for TDS in the literature.

\section{Overview of LAGUERRE-BASEd Model ORder REDUCTION}

Consider a multiple-input-multiple-output (MIMO) descriptor system of the form

$$
\begin{aligned}
\mathbf{E} \dot{x}(t) & =\mathbf{A} x(t)+\mathbf{B} u(t) \\
y(t) & =\mathbf{C} x(t)+\mathbf{D} u(t)
\end{aligned}
$$

with (McMillan) degree $n$ and number of ports $p$. $\mathbf{A}$ and $\mathbf{E}$ are respectively known as the state and descriptor matrices. The dimensions of the matrices $\mathbf{A}, \mathbf{B}, \mathbf{C}, \mathbf{D}, \mathbf{E}$ are respectively $n \times n, n \times p, p \times n, p \times p$ and $n \times n$.

The transfer function of this system is

$$
\mathbf{H}(s)=\mathbf{C}(s \mathbf{E}-\mathbf{A})^{-1} \mathbf{B}+\mathbf{D} .
$$

Note that it is usually required that $s \mathbf{E}-\mathbf{A}$ is a regular matrix pencil [24].

The $i^{\text {th }}$ Laguerre polynomial is defined as

$$
\mathcal{L}_{i}(t)=\frac{e^{t}}{i !} \frac{d^{i}}{d t^{i}}\left(e^{-t} t^{i}\right)
$$

and the scaled Laguerre functions are

$$
\Phi_{i}^{\alpha}(t)=\sqrt{2 \alpha} e^{-\alpha t} \mathcal{L}_{i}(2 \alpha t) i=0,1 \ldots
$$

Here $\alpha$ is a positive scaling parameter called the Laguerre parameter or time-scale factor [25]

In the Laplace domain, the transformation of the scaled Laguerre functions can be written as

$$
\Phi_{i}^{\alpha}(s)=\frac{\sqrt{2 \alpha}}{s+\alpha}\left(\frac{s-\alpha}{s+\alpha}\right)^{i} i=0,1 \ldots
$$

In [21], it has been shown that the transfer function of a strictly proper $(\mathbf{D}=0)$ stable system can be expanded into the Laguerre orthonormal basis $\Phi_{i}^{\alpha}(s)$ as

$$
\mathbf{H}(s)=\mathbf{C}(s \mathbf{E}-\mathbf{A})^{-1} \mathbf{B}=\frac{\sqrt{2 \alpha}}{s+\alpha} \sum_{i=0}^{\infty} \mathbf{F}_{i}\left(\frac{s-\alpha}{s+\alpha}\right)^{i}
$$

where the $\mathbf{F}_{i}$ 's are the Laguerre coefficients, which can be matrices in general. Applying the bilinear transformation (7) to the r.h.s of formula (6),

$$
s=\alpha \frac{1+u}{1-u}
$$

we obtain

$$
\mathbf{C}(u(\alpha \mathbf{E}+\mathbf{A})-(\mathbf{A}-\alpha \mathbf{E}))^{-1} \mathbf{B}=\frac{1}{\sqrt{2 \alpha}} \sum_{i=0}^{\infty} \mathbf{F}_{i} u^{i} .
$$

From this it can be inferred that the $q$ th-order Padé approximation of the modified transfer matrix

$$
\hat{\mathrm{H}}(u)=\mathbf{C}(u(\alpha \mathbf{E}+\mathbf{A})-(\mathbf{A}-\alpha \mathbf{E}))^{-1} \mathbf{B}
$$

in the $u$-domain is equivalent to the $q$ th-order Laguerre approximation in the $s$-domain.

Note that the bilinear transformation (6) maps the Laplace $s$-domain onto the Laguerre $u$-domain. It is seen, by comparing (9) and (2) that the state-space matrices have been modified to $\mathbf{E}_{u}=\alpha \mathbf{E}+\mathbf{A}$ and $\mathbf{A}_{u}=\mathbf{A}-\alpha \mathbf{E}$.

Thus, on defining $\mathbf{G}=\mathbf{A}_{u}^{-1} \mathbf{E}_{u}$ and $\mathbf{L}=\mathbf{A}_{u}^{-1} \mathbf{B}$, for a reduced order $q$ the $n \times q$ modified Krylov subspace $\mathscr{K}_{q}$ [26], [27] is:

$$
\mathscr{K}_{q}(\mathbf{G}, \mathbf{L})=\text { colspan }\left[\mathbf{L}, \mathbf{G L}, \mathbf{G}^{\mathbf{2}} \mathbf{L}, \ldots, \mathbf{G}^{\mathbf{q}-\mathbf{1}} \mathbf{L}\right]
$$

This yields a reduced order system described by

$$
\begin{aligned}
\mathbf{A}_{r} & =\mathbf{Q}^{T} \mathbf{A} \mathbf{Q} \\
\mathbf{E}_{r} & =\mathbf{Q}^{T} \mathbf{E} \mathbf{Q} \\
\mathbf{B}_{r} & =\mathbf{Q}^{T} \mathbf{B} \\
\mathbf{C}_{r} & =\mathbf{C Q} .
\end{aligned}
$$

Here the column-orthogonal matrix $\mathbf{Q}$ is found through the singular value decomposition (SVD) approach on the Krylov subspace $\mathscr{K}_{q}$ (10) i.e;

$$
\mathbf{U} \Sigma \mathbf{V}^{T}=\operatorname{SVD}\left(\mathscr{K}_{q}(\mathbf{G}, \mathbf{L})\right)
$$

where $\mathbf{U}$ is the column-orthogonal matrix, $\Sigma$ is a diagonal matrix containing the singular values and $\mathbf{V}$ is orthogonal matrix of dimensions $N \times q, q \times q$ and $q \times q$ respectively, $q$ is the reduced order.

Thus $\mathbf{Q}$ is equal to the left SVD column-orthogonal factor 
Note that the algorithm makes use of the Laguerre functions, but they do not appear in the algorithm. The main aim of using the Laguerre functions is to get rid of the $s$ term in $(s \mathbf{E}-\mathbf{A})^{-1}(2)$. Instead the algorithm makes use of the inverse of $\mathbf{A}_{u}=(\mathbf{A}-\alpha \mathbf{E})$. The parameter $\alpha$ is chosen such that the inverse exists.

\section{PROPOSED ALGORITHM}

Consider a time-delay system of degree $n$ with $p$ ports having $k$ delays $\tau_{j}$, present in both the state and descriptor matrices, which can be represented in general delayed state space form as:

$$
\begin{aligned}
\mathbf{E}_{\mathbf{0}} \dot{x}(t)+ & \sum_{j=1}^{k} \mathbf{E}_{\mathbf{j}} \dot{x}\left(t-\tau_{j}\right)= \\
& \mathbf{A}_{\mathbf{0}} x(t)+\sum_{j=1}^{k} \mathbf{A}_{\mathbf{j}} x\left(t-\tau_{j}\right)+\mathbf{B} u(t) \\
y(t)= & \mathbf{C} x(t)+\mathbf{D} u(t) .
\end{aligned}
$$

Here, $x(t) \in \mathscr{R}^{n}$ is the state vector; $u(t) \in \mathscr{R}^{p}$ is the control input with $u(t)=0$ for $t<0 ; y(t) \in \mathscr{R}^{p}$ is the output. $\mathbf{A}_{\mathbf{0}}, \mathbf{A}_{\mathbf{j}}, \mathbf{E}_{\mathbf{0}}, \mathbf{E}_{\mathbf{j}}, \mathbf{B}, \mathbf{C}, \mathbf{D}$ are constant matrices with appropriate dimensions. From (13) we obtain the transfer function $\mathbf{H}(s)$ as:

$$
\begin{aligned}
\mathbf{H}(s) & =\mathbf{C}\left(s\left(\mathbf{E}_{\mathbf{0}}+\sum_{j=1}^{k} \mathbf{E}_{j} e^{-s \tau_{j}}\right)\right. \\
& \left.-\left(\mathbf{A}_{\mathbf{0}}+\sum_{j=1}^{k} \mathbf{A}_{j} e^{-s \tau_{j}}\right)\right)^{-1} \mathbf{B}+\mathbf{D} .
\end{aligned}
$$

\section{A. Dealy Approximation}

The delay terms $e^{-s \tau_{j}}$ in (14) are mapped onto the Laguerre domain by applying the bilinear transformation (7). Thus obtaining:

$$
e^{-s \tau_{j}}=e^{-a_{j}\left(\frac{1+u}{1-u}\right)}
$$

where $a_{j}=\alpha \tau_{j}$. As the generating function of the Laguerre polynomial [28] [29] is given by,

$$
\frac{e^{\frac{-x t}{(1-t)}}}{(1-t)}=\sum_{i=0}^{\infty} \mathcal{L}_{i}(x) t^{i}
$$

we can easily prove that

$e^{-a_{j}\left(\frac{1+u}{1-u}\right)}=e^{-a_{j}} e^{\left(-2 a_{j}\left(\frac{u}{1-u}\right)\right)}=e^{-a_{j}}(1-u) \sum_{i=0}^{\infty} \mathcal{L}_{i}\left(2 a_{j}\right) u^{i}$.

Using the recurrence relation of Laguerre polynomials, we obtain the approximation series in powers of $u$ as shown,

$$
e^{-a_{j}\left(\frac{1+u}{1-u}\right)}=e^{-a_{j}}+\sum_{i=1}^{\infty} e^{-a_{j}}\left(\mathcal{L}_{i}\left(2 a_{j}\right)-\mathcal{L}_{i-1}\left(2 a_{j}\right)\right) u^{i} .
$$

This will help us to obtain a suitable form for the transfer function of the TDSs in $u$ domain for deriving the higher-order Krylov.
The delay term is approximated to an order $r$ i.e;

$$
e^{-a_{j}\left(\frac{1+u}{1-u}\right)} \approx T_{d}^{r}=e^{-a_{j}}+\sum_{i=1}^{r} e^{-a_{j}}\left(\mathcal{L}_{i}\left(2 a_{j}\right)-\mathcal{L}_{i-1}\left(2 a_{j}\right)\right) u^{i} .
$$

Such that the error is below a defined threshold as shown

$$
\left\|e^{-a_{j}\left(\frac{1+u}{1-u}\right)}-T_{d}^{r}\right\|_{2} \leq \text { threshold } .
$$

The threshold is set to a desired level of accuracy required for the reduced order model.

Then (14) is mapped to the $u$-domain similar to that of (9), in which the approximated delay term (18) is substituted to obtain an accurate reduced order TDS as described in the following section.

\section{B. Reduced Order Estimation}

A priori reduced order estimation makes the construction of the reduced order TDS much more efficient. The reduced order can be estimated by studying the Hankel singular values (HSVs) of the system. The HSVs provides a measure of energy for each state in a system [30].

1) Zero-order Approximation Technique (ZAT): To obtain an initial estimation for the reduced order of the TDSs we consider a zero order approximation for the delay term i.e;

$$
e^{-a_{j}\left(\frac{1+u}{1-u}\right)} \approx e^{-a_{j}}
$$

Thus, approximating the system matrices of (13) with delay as:

$$
\begin{aligned}
& \mathbf{E}_{Z A T}(u)=\mathbf{E}_{\mathbf{0}}+\sum_{j=1}^{k} \mathbf{E}_{j}\left(e^{-a_{j}\left(\frac{1+u}{1-u}\right)}\right) \approx \mathbf{E}_{\mathbf{0}}+\sum_{j=1}^{k} \mathbf{E}_{j}\left(e^{-a_{j}}\right) \\
& \mathbf{A}_{Z A T}(u)=\mathbf{A}_{\mathbf{0}}+\sum_{j=1}^{k} \mathbf{A}_{j}\left(e^{-a_{j}\left(\frac{1+u}{1-u}\right)}\right) \approx \mathbf{A}_{\mathbf{0}}+\sum_{j=1}^{k} \mathbf{A}_{j}\left(e^{-a_{j}}\right)
\end{aligned}
$$

From the decay rate of the HSVs of the system $\left(\mathbf{A}_{Z A T}, \mathbf{B}, \mathbf{C}\right.$, $\left.\mathbf{D}, \mathbf{E}_{Z A T}\right)$, the reduced order $q$ can be estimated [31]. Then the reduced order is increased from $q$ by a bottom-up approach depending on the required pre-defined accuracy.

\section{Higher-Order Laguerre Approximation Technique (HLAT)}

After estimating the reduced order, the transfer function (14) of the strictly proper TDS is then written in terms of Laguerre expansion similar to that of (9), i.e. by mapping $s$-domain to $u$-domain

$$
\mathbf{H}(u)=\mathbf{C}\left(\alpha\left(\frac{1+u}{1-u}\right) \mathbf{E}_{T D S}(u)-\mathbf{A}_{T D S}(u)\right)^{-1} \mathbf{B}
$$

Then, (18) is substituted for the delay term in (22) such that (21) becomes: 


$$
\begin{aligned}
& \mathbf{E}_{T D S}(u)=\mathbf{E}_{\mathbf{0}}+\sum_{j=1}^{k} \mathbf{E}_{j}\left(e^{-a_{j}}\right) \\
& \left(1+\sum_{i=1}^{r} e^{-a_{j}}\left(\mathcal{L}_{i}\left(2 a_{j}\right)-\mathcal{L}_{i-1}\left(2 a_{j}\right)\right) u^{i}\right) \\
& \mathbf{A}_{T D S}(u)=\mathbf{A}_{\mathbf{0}}+\sum_{j=1}^{k} \mathbf{A}_{j}\left(e^{-a_{j}}\right) \\
& \left(1+\sum_{i=1}^{r} e^{-a_{j}}\left(\mathcal{L}_{i}\left(2 a_{j}\right)-\mathcal{L}_{i-1}\left(2 a_{j}\right)\right) u^{i}\right) .
\end{aligned}
$$

On substituting (23) in (22) we can write the modified transfer function in $u$-domain as,

$$
\hat{\mathrm{H}}(u)=\mathbf{C}\left(\phi_{r+1} u^{r+1}+\phi_{r} u^{r}+\ldots+\phi_{1} u+\phi_{0}\right)^{-1} \mathbf{B} .
$$

Here $\phi_{i}$ for $i=0,1, \ldots, r+1$ are the coefficients of $i^{t h}$ power of $u$, where the constant term

$$
\phi_{0}=\alpha\left(\mathbf{E}_{\mathbf{0}}+\sum_{j=1}^{k} \mathbf{E}_{j}\left(e^{-a_{j}}\right)\right)-\left(\mathbf{A}_{\mathbf{0}}+\sum_{j=1}^{k} \mathbf{A}_{j}\left(e^{-a_{j}}\right)\right),
$$

the coefficient of $u$

$$
\begin{aligned}
\phi_{1} & =\alpha\left(\mathbf{E}_{\mathbf{0}}+\sum_{j=1}^{k} \mathbf{E}_{j}\left(e^{-a_{j}}\right)\left(1-2 a_{j}\right)\right) \\
& +\mathbf{A}_{\mathbf{0}}+\sum_{j=1}^{k} \mathbf{A}_{j}\left(e^{-a_{j}}\right)\left(1+2 a_{j}\right),
\end{aligned}
$$

the coefficient of $u^{i}$ for $i=2, \ldots, r$

$$
\begin{aligned}
\phi_{i} & =\alpha \sum_{j=1}^{k} \mathbf{E}_{j}\left(e^{-a_{j}}\left(\mathcal{L}_{i}\left(2 a_{j}\right)-\mathcal{L}_{i-2}\left(2 a_{j}\right)\right)\right. \\
& -\sum_{j=1}^{k} \mathbf{A}_{j}\left(e^{-a_{j}}\left(\mathcal{L}_{i}\left(2 a_{j}\right)-2 \mathcal{L}_{i-1}\left(2 a_{j}\right)+\mathcal{L}_{i-2}\left(2 a_{j}\right)\right)\right.
\end{aligned}
$$

and the coefficient of $u^{r+1}$

$$
\phi_{r+1}=\sum_{j=1}^{k}\left(\alpha \mathbf{E}_{j}+\mathbf{A}_{j}\right)\left(e^{-a_{j}}\left(\mathcal{L}_{r}\left(2 a_{j}\right)-\mathcal{L}_{r-1}\left(2 a_{j}\right)\right) .\right.
$$

Note that different order of approximation can be chosen for each delay thereby the coefficients of $u$ will change accordingly for (24). In this paper the order of approximation is considered equal for all the delays and is equal to that of the largest delay in the TDSs so that the accuracy is guaranteed.

In [32], [33], moment matching theorems for Krylov subspace based model reduction of higher order linear dynamical systems are presented in the context of higher order Krylov subspaces.

For a transfer function of the form,

$$
\mathbf{H}(s)=\mathbf{C}\left(\sigma_{r} s^{r}+\sigma_{r-1} s^{r-1}+\ldots+\sigma_{1} s+\sigma_{0}\right)^{-1} \mathbf{B} .
$$

The $r$ th order Krylov subspace is defined as

$$
\mathscr{K}_{q}\left(\mathbf{G}_{1}, \mathbf{G}_{2}, \ldots, \mathbf{G}_{r}, \mathbf{L}\right)=\text { colspan }\left[P_{0}, P_{1}, \ldots, P_{q-1}\right],
$$

where $\mathbf{L}=\sigma_{0}^{-1} \mathbf{B}$ and $\mathbf{G}_{i}=\sigma_{0}^{-1} \sigma_{i}$ for $i=1,2, \ldots, r$ and

$$
\begin{aligned}
P_{0} & =\mathbf{L} ; \quad P_{i}=0 \text { for } i<0 \\
P_{i} & =\mathbf{G}_{1} P_{i-1}+\ldots+\mathbf{G}_{r} P_{i-r},
\end{aligned}
$$

This subspace is a generalization of Krylov subspaces for higher order systems and eliminates the linearization step of rewriting the higher-order system in an equivalent first-order form to prove moment-matching properties. However, to match the moments of an $\mathrm{r}$-th order model, the matrix $\sigma_{0}$ should be invertible. Thus, (24) can be inferred as Padé approximation of the modified transfer matrix in the $u$-domain.

Thereby, the higher order Krylov subspace $\mathscr{K}_{q}$ is defined as in (30), with $\mathbf{L}=\phi_{0}^{-1} \mathbf{B}$ and $\mathbf{G}_{i}=\phi_{0}^{-1} \phi_{i}$ for $i=1,2, \ldots, r+1$. The column-orthogonal matrix $\mathbf{Q}$ for congruence transformation is found through the singular value decomposition (SVD) approach i.e;

$$
\mathbf{U} \Sigma \mathbf{V}^{T}=\operatorname{SVD}\left(\mathscr{K}_{q}\left(\mathbf{G}_{1}, \mathbf{G}_{2}, \ldots, \mathbf{G}_{r}, \mathbf{L}\right)\right)
$$

Thus $\mathbf{Q}$ is equal to the left SVD column-orthogonal factor $\mathbf{U}$ of dimension $n \times q$ associated with the $(r+1)^{t h}$ Krylov subspace.

The column-orthogonal matrix $\mathbf{Q}$ thus yields reduced order state-space matrices through congruence transformation described by

$$
\begin{array}{cll}
\mathbf{A}_{\mathbf{0} r}=\mathbf{Q}^{T} \mathbf{A}_{\mathbf{0}} \mathbf{Q} & , & \mathbf{A}_{\mathbf{j}_{r}}=\mathbf{Q}^{T} \mathbf{A}_{\mathbf{j}} \mathbf{Q} \\
\mathbf{E}_{\mathbf{0} r}=\mathbf{Q}^{T} \mathbf{E}_{\mathbf{0}} \mathbf{Q} & , & \mathbf{E}_{\mathbf{j}_{r}}=\mathbf{Q}^{T} \mathbf{E}_{\mathbf{j}} \mathbf{Q} \\
\mathbf{B}_{r}=\mathbf{Q}^{T} \mathbf{B} & , & \mathbf{C}_{r}=\mathbf{C Q},
\end{array}
$$

such that the reduced-order transfer matrix $\mathbf{H}_{r}(s)$ is

$$
\begin{aligned}
\mathbf{H}_{r}(s) & =\mathbf{C}_{r}\left(s\left(\mathbf{E}_{\mathbf{0}_{r}}+\sum_{j=1}^{k} \mathbf{E}_{\mathbf{j}_{r}} e^{-s \tau_{j}}\right)\right. \\
& \left.-\left(\mathbf{A}_{\mathbf{0}_{r}}+\sum_{j=1}^{k} \mathbf{A}_{\mathbf{j}_{r}} e^{-s \tau_{j}}\right)\right)^{-1} \mathbf{B}_{r}
\end{aligned}
$$

The algorithmic steps of the proposed techniques is shown as a flowchart in Fig. 1.

Concerning the complexity of the proposed technique, it can be noted that the most expensive step is related to ZAT for an initial guess of the reduced order and its complexity is $O\left(n^{3}\right)$ with n equal to the actual order of the system. If the order of the system $n$ is larger than 3000, then its advisable to estimate the order using the bottom-up approach. Next, the higher-order Krylov subspace is computed and the SVD has to be performed to obtain the column-orthogonal matrix $Q$ (32), which has a complexity of $O\left(4 n^{2} q\right)$ where $q$ is the column size of the $Q$ matrix.

\section{Numerical Results}

The numerical simulations were performed on a Windows 7 platform on Intel ${ }^{(R)} \mathrm{Core}^{(T M)} 2$ Duo P8700 $2.53 \mathrm{GHz}$ machine with 2 GB RAM and has been implemented in Matlab R2010a.

\section{Error criteria}

The reduced order estimation using ZAT as described in Section III-A depends on the pre-defined accuracy required. 


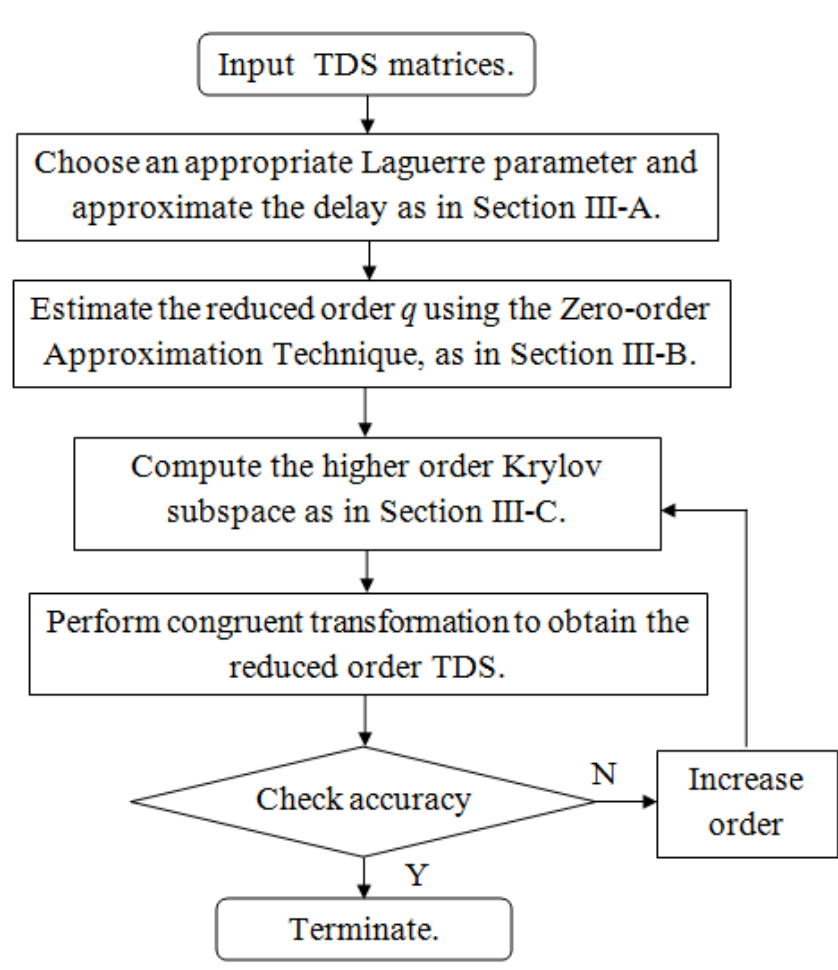

Fig. 1. Flowchart of the proposed algorithm.

For this paper, the weighted RMS error is used to assess the accuracy of the reduced order model with a target accuracy of 0.001 .

The weighted RMS error between the original frequency response $H_{i j}$ and the reduced order model $H_{r,(i j)}$ is defined as:

$$
\begin{gathered}
E r r=\sqrt{\frac{\sum_{k=1}^{K_{s}} \sum_{i=1}^{P_{i n}} \sum_{j=1}^{P_{\text {out }}} \frac{\left|H_{r,(i j)}\left(s_{k}\right)-H_{(i j)}\left(s_{k}\right)\right|^{2}}{W_{(i j)}\left(s_{k}\right)}}{P_{\text {in }} P_{\text {out }} K_{s}}} \\
W_{(i j)}\left(s_{k}\right)=\left|H_{(i j)}\left(s_{k}\right)\right|^{2} .
\end{gathered}
$$

In (35) $K_{S}, P_{\text {in }}$ and $P_{\text {out }}$ are the number of frequency samples, input and output ports of the system, respectively.

The results obtained using the proposed technique is validated by comparing with Augmented MOR [5] and multiorder Arnoldi MOR [13].

\section{A. Backplane Vias (BPV)}

A simple backplane via is considered [34] which consists of a probe launch, the differential via, which includes the through part and the stub part, and the uniform stripline section. A simple topology that includes these features is shown in Fig. 2. Each probe launch is composed of four uniform transmission line elements which is modeled using the conventional lumped model as in [21]. The via model is the simplest possible model and the uniform stripline structure is modeled using the method of characteristics [5] as a simple, dual lossless stripline based on the geometry as described in [34], [35].

Then the TDSs is constructed as a linear interconnect network with a set of TLs as elaborated in [5]. For the modeling, the per-unit-length (PUL) parameter matrices of the TLs are extracted utilizing the approaches described in [1].

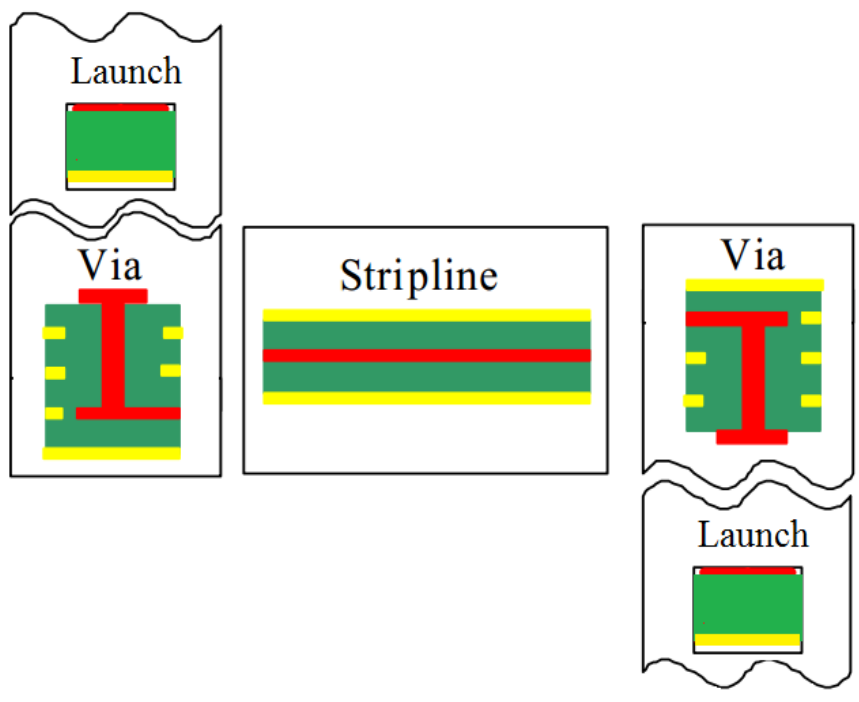

Fig. 2. BPV: Block Diagram of backplane vias.

The order of the original model is 1448 with $\tau_{\max }=0.057 \mathrm{~ns}$. The Laguerre parameter $\alpha$ for the proposed technique is $38 \pi e 9$ and the expansion point for the comparison technique is set to half of the maximum frequency.

Fig. 3 plots the HSVs of the approximated TDS, from which an initial guess for the reduced order of the TDSs is obtained.

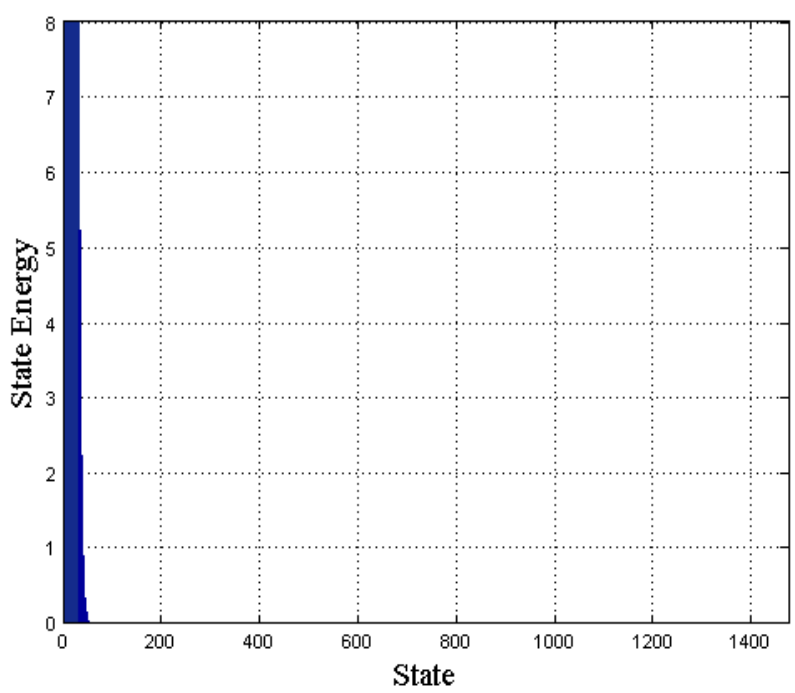

Fig. 3. BPV: Hankel Singular Values (state contributions) of the approximated model using ZAT.

Fig. 4 and Fig. 5 plots the magnitude and phase of $S_{14}(s)$ of the original model with reduced order models of order 387 obtained using the proposed technique.

Table I compares the efficiency of the different techniques for achieving a weighted RMS error (35) smaller than 0.001 with 201 frequency samples in the range [1-10] GHz. For both the techniques the delays are approximated to an order 


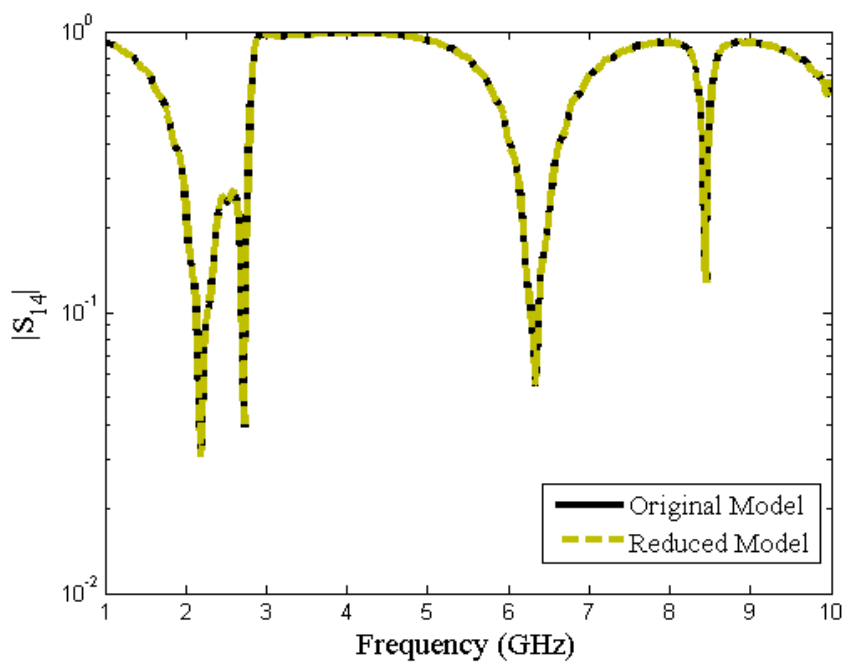

Fig. 4. BPV: Magnitude of $S_{14}$.

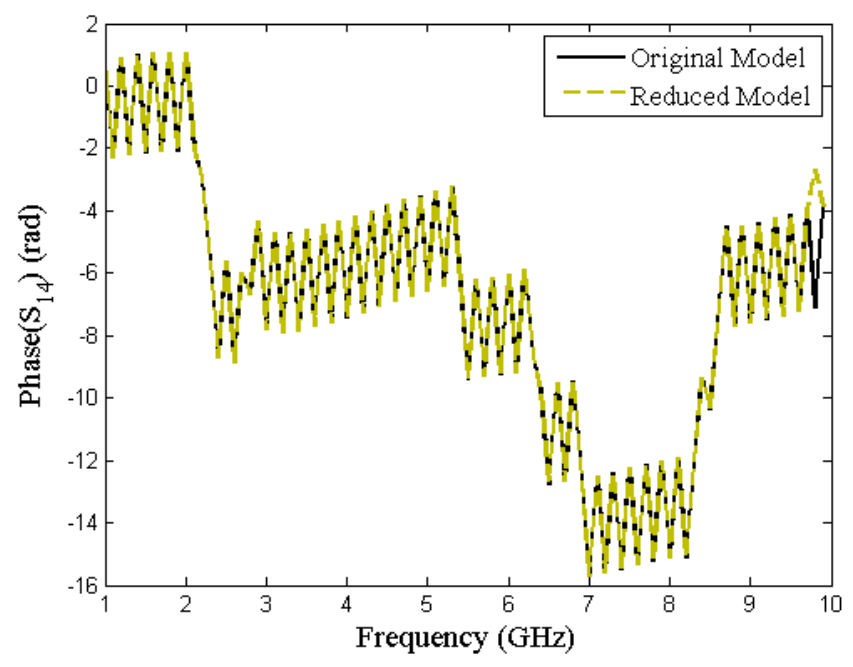

Fig. 5. BPV: Phase of $S_{14}$.

of 7 and is obtained by setting a threshold of 0.01 for (19). If we consider a higher threshold then we might not be able to obtain an accuracy of 0.001 for the reduced order TDSs. Fig. 6 shows the approximation curve for $\tau_{\max }=0.057 \mathrm{~ns}$ considering $\mathrm{r}=2,5,7$.

In Table I the CPU time for the proposed technique includes the time for the reduced order estimation and the time for generating the reduced TDSs, while that for the multiorder Arnoldi is with a bottom-up approach for generating the reduced order TDSs with the required accuracy. The technique presented in [5] using augmented first order systems could not be used for the comparison due to memory limitations.

Table II compares the total size and simulation times of the original and proposed algorithm. For this example, the simulation time of the reduced order TDSs is about 388 times faster when compared to the original system.

Thus the proposed method is more efficient, and it also
TABLE I

BPV: EFFICIENCY COMPARISON FOR ACHIEVING A WEIGHTED RMS ERROR OF 0.001

\begin{tabular}{|l|l|l|}
\hline Technique & Multiorder Arnoldi [13] & Proposed \\
\hline Initial Order & 1448 & 1448 \\
Order of delay approximation & 7 & 7 \\
Reduced Order & 388 & 387 \\
CPU time (sec) & 897.7 & 702.2 \\
\hline
\end{tabular}

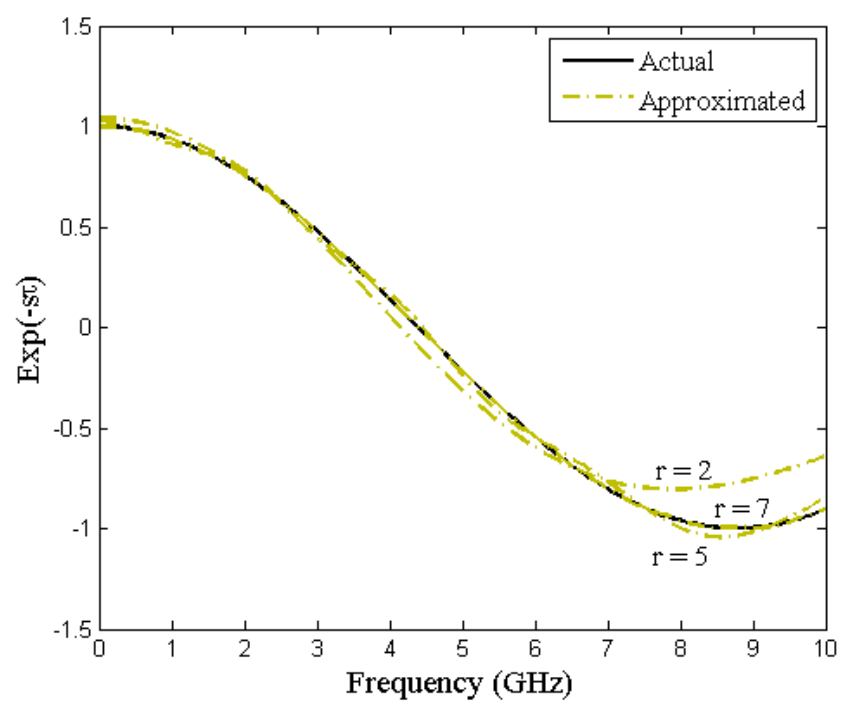

Fig. 6. BPV: Approximation of the delay term.

provides a compact model with the Laguerre expansion.

\section{B. RLC Network With Delay Elements}

A system consisting of two lossless multiconductor transmission lines (MTLs) and three RLC networks has been modeled ( see Fig. 7). The detailed procedures of constructing such TDSs are elaborated in [5]. Each MTL block is composed of three conductors and it has a length equal to $2 \mathrm{~cm}$ in total and the RLC network has a length of $1.5 \mathrm{~cm}$ in total.

The order of the original network is 2625 with $\tau_{\max }=$ $0.02 n s$. The frequency range of interest for this system is [0-6] GHz. The Laguerre parameter $\alpha$ for the proposed method is $24 \pi e 9$ and the expansion point for the compared techniques is set to half of the maximum frequency.

The reduced order is estimated as described in Section III-B, from the HSVs of the approximated TDSs.

Fig. 9 - Fig 10 compares the magnitude and phase of the original and Laguerre-based reduced delayed model $Y_{14}(s)$. A good agreement is obtained between the original and reduced model.

Table III compares the efficiency of the different techniques for achieving a weighted RMS error (35) smaller than 0.001 with 201 frequency samples in the range [0-6] GHz. As in the former example for a threshold of 0.01 for (19), the order of approximation for the delay is 5 for both the techniques. 
TABLE II

BPV: COMPUTATIONAL INFORMATION

\begin{tabular}{|l|l|l|l|}
\hline Solution method & Size & $\begin{array}{l}\text { CPU Time for } \\
\text { generating the re- } \\
\text { duced TDSs }\end{array}$ & Simulation Time \\
\hline $\begin{array}{l}\text { Original System } \\
\text { Proposed }\end{array}$ & 1448 & & 2406.82 \\
\hline
\end{tabular}

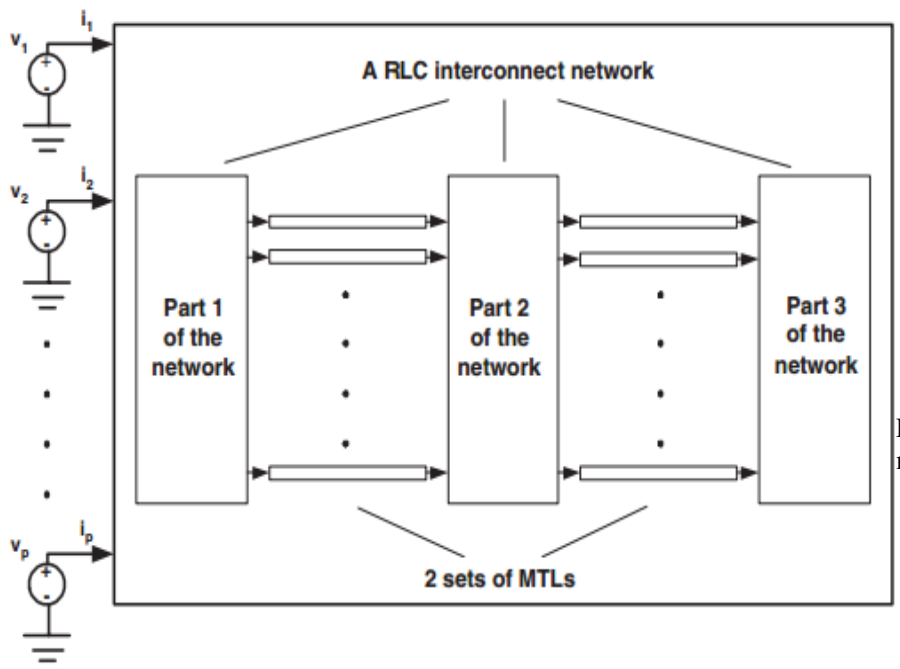

Fig. 7. Schematic of RLC network including delay elements.

The CPU time for the proposed technique includes the time for the reduced order estimation and the time for generating the reduced TDSs, while that for the multiorder Arnoldi is with a bottom-up approach for generating the reduced order TDSs with the required accuracy. The technique presented in [5] using augmented first order systems could not be used for the comparison due to memory limitations.

TABLE III

RLC: EFFICIENCY COMPARISON FOR ACHIEVING A WEIGHTED RMS ERROR OF 0.001

\begin{tabular}{|l|l|l|}
\hline Technique & Multiorder Arnoldi [13] & Proposed \\
\hline Initial Order & 2625 & 2625 \\
Order of delay approximation & 5 & 5 \\
Reduced Order & 212 & 213 \\
CPU time (sec) & 934.70 & 896.73 \\
\hline
\end{tabular}

TABLE IV

RLC: COMPUTATIONAL INFORMATION

\begin{tabular}{|l|l|l|l|}
\hline Solution method & Size & $\begin{array}{l}\text { CPU Time for } \\
\text { generating the re- } \\
\text { duced TDSs }\end{array}$ & Simulation Time \\
\hline $\begin{array}{l}\text { Original System } \\
\text { Proposed }\end{array}$ & $\begin{array}{l}2625 \\
213\end{array}$ & 896.73 & 3409.42 \\
\hline
\end{tabular}

Similar to the previous example Table IV compares the total size and simulation times of the original and proposed

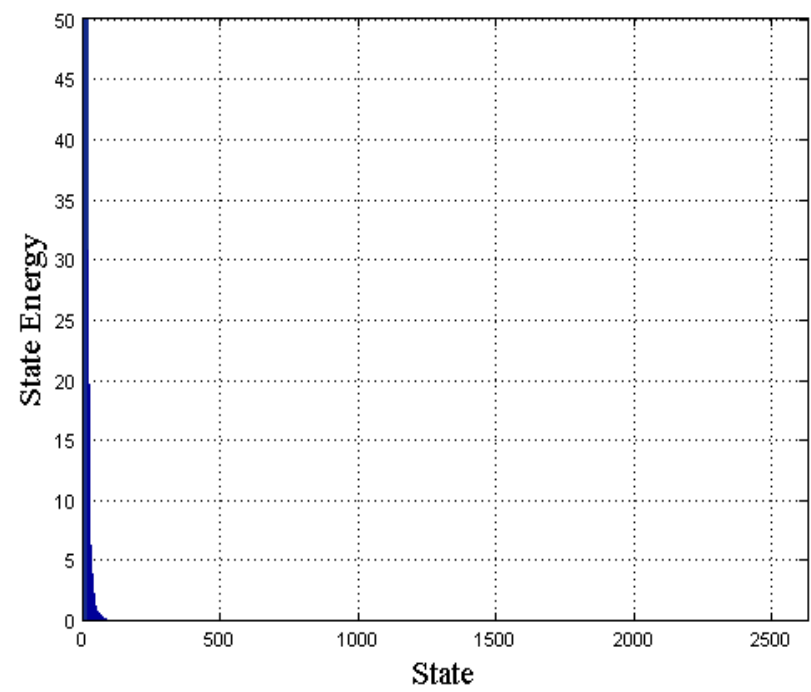

Fig. 8. RLC: Hankel Singular Values (state contribution) of the approximated model using ZAT.

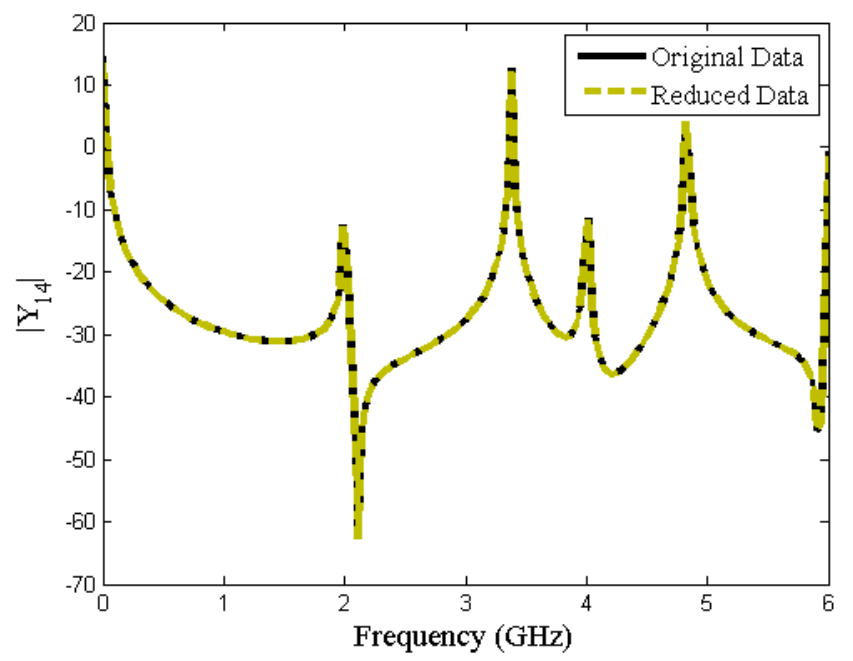

Fig. 9. RLC: Magnitude of $Y_{14}$.

algorithm and the simulation time of the reduced order TDSs is about 980 times faster when compared to the original system.

Thus, from the above results we see that the proposed technique is able to reduce TDS more efficiently to achieve the required accuracy using Laguerre expansion.

\section{CONClusion}

In this paper, we have presented a novel model order reduction method for large linear networks that contain delay elements. The algorithm is based on higher order Krylov subspace for model order reduction of time-delay systems based on a Laguerre expansion technique.The proposed technique consists of two parts: 1) the delays are approximated using recursive relation of Laguerre polynomial then 2) the reduced order is estimated for the time-delay system using a smart 


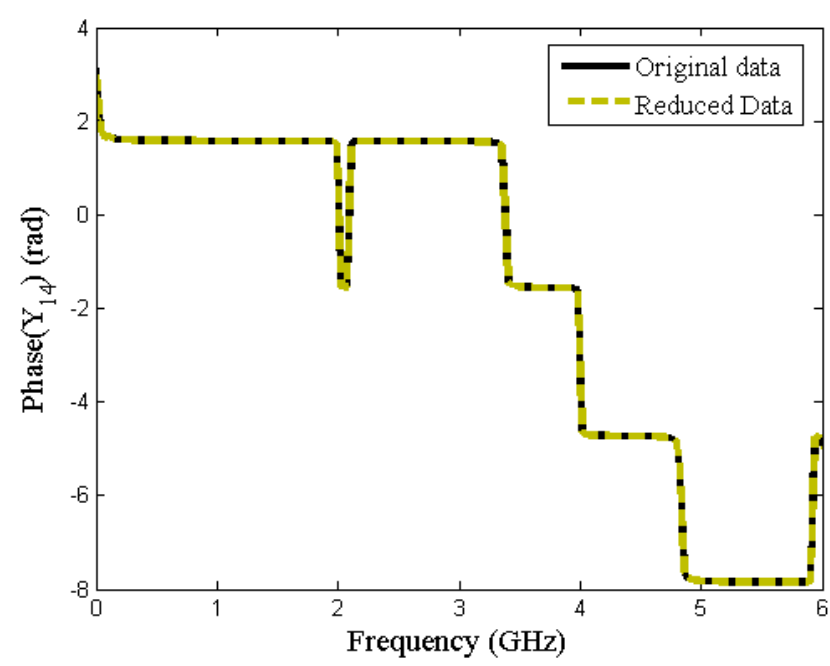

Fig. 10. RLC: Phase of $Y_{14}$.

truncation of the delay term and 3) a higher order Krylov subspace is computed using Laguerre expansion for obtaining the congruence-based reduced order time-delay system. The proposed technique is validated by real world examples for its efficiency and accuracy.

/

\section{REFERENCES}

[1] C. Paul, Analysis of Multiconductor Transmission Lines. New York: Wiley, 1994.

[2] R. Achar and M. Nakhla, "Simulation of high-speed interconnects," Proceedings of the IEEE, vol. 89, no. 5, pp. 693-728, May 2001.

[3] T. Dhaene and D. de Zutter, "Selection of lumped element models for coupled lossy transmission lines," IEEE Transactions on ComputerAided Design of Integrated Circuits and Systems,, vol. 11, no. 7, pp. 805 -815, Jul. 1992.

[4] J.-P. Richard, "Time-delay systems: an overview of some recent advances and open problems," Automatica, vol. 39, no. 10, pp. 1667 1694, 2003.

[5] W. Tseng, C. Chen, E. Gad, M. Nakhla, and R. Achar, "Passive order reduction for RLC circuits with delay elements," IEEE Transactions on Advanced Packaging, vol. 30, no. 4, pp. 830 -840, Nov. 2007.

[6] E. Chiprout and M. Nakhla, "Analysis of interconnect networks using complex frequency hopping (CFH)," IEEE Transactions on ComputerAided Design of Integrated Circuits and Systems, vol. 14, no. 2, pp. 186 -200 , Feb. 1995.

[7] T. Tang and M. Nakhla, "Analysis of high-speed vlsi interconnects using the asymptotic waveform evaluation technique," IEEE Transactions on Computer-Aided Design of Integrated Circuits and Systems, vol. 11, no. 3, pp. $341-352$, Mar. 1992.

[8] P. Feldmann and R. Freund, "Efficient linear circuit analysis by pade approximation via the lanczos process," IEEE Transactions on ComputerAided Design of Integrated Circuits and Systems, vol. 14, no. 5, pp. 639 -649, May 1995.

[9] P. Gunupudi, M. Nakhia, and R. Achar, "Simulation of high-speed distributed interconnects using krylov-space techniques," IEEE Transactions on Computer-Aided Design of Integrated Circuits and Systems, vol. 19 , no. 7 , pp. $799-808$, Jul 2000.

[10] A. Charest, M. Nakhla, and R. Achar, "Passive model-order reduction of RLC circuits with embedded time-delay descriptor systems," IEEE 20th Conference on Electrical Performance of Electronic Packaging and Systems, pp. $223-226$, Oct. 2011.

[11] A. Charest, M. Nakhla, R. Achar, and D. Saraswat, "Passivity verification of delayed rational function based macromodels of tabulated networks characterized by scattering parameters," IEEE Transactions on Components, Packaging and Manufacturing Technology, vol. 1, no. 3, pp. $386-398$, Mar. 2011.
[12] F. Ferranti, M. Nakhla, G. Antonini, T. Dhaene, L. Knockaert, and A. Ruehli, "Multipoint full-wave model order reduction for delayed PEEC models with large delays," IEEE Transactions on Electromagnetic Compatibility, vol. 53, no. 4, pp. 959 -967, Nov. 2011.

[13] E. Rasekh and A. Dounavis, "Multiorder arnoldi approach for model order reduction of PEEC models with retardation," IEEE Transactions on Components, Packaging and Manufacturing Technology, vol. 2, no. 10 pp. $1629-1636$, Oct. 2012.

[14] G. Kequin, K. L. Vladimir, and C. Jie, Stability of Time-Delay Systems. New York: Birkhauser Boston, 2003.

[15] W. Michiels, E. Jarlebring, and K. Meerbergen, "Krylov-based model order reduction of time-delay systems," SIAM J. Matrix Anal. Appl., vol. 32, no. 4, pp. 1399-1421, 2011.

[16] J. Phillips, E. Chiprout, and D. Ling, "Efficient full-wave electromagnetic analysis via model-order reduction of fast integral transforms," 33rd Design Automation Conference Proceedings 1996, pp. 377 -382, Jun., 1996.

[17] J. Cullum, A. Ruehli, and T. Zhang, "A method for reduced-order modeling and simulation of large interconnect circuits and its application to PEEC models with retardation," IEEE Transactions on Circuits and Systems II: Analog and Digital Signal Processing, vol. 47, no. 4, pp. $261-273$, Apr. 2000.

[18] Y. Su, J. Wang, X. Zeng, Z. Bai, C. Chiang, and D. Zhou, "SAPOR: second-order arnoldi method for passive order reduction of rcs circuits," IEEE/ACM International Conference on Computer Aided Design, 2004. ICCAD-2004., pp. 74 - 79, Nov. 2004.

[19] Z. Bai and Y. Su, "Dimension reduction of large-scale second-order dynamical systems via a second-order arnoldi method," SIAM Journal on Scientific Computing, vol. 26, no. 5, pp. 1692-1709, 2005.

[20] P. M. Mäkilä, "Laguerre series approximation of infinite dimensional systems," Automatica, vol. 26, no. 6, pp. 985 - 995, 1990.

[21] L. Knockaert and D. De Zutter, "Laguerre-SVD reduced-order modeling," IEEE Transactions on Microwave Theory and Techniques, vol. 48, no. 9, pp. $1469-1475$, Sept. 2000

[22] X.-L. Wang and Y.-L. Jiang, "Model order reduction methods for coupled systems in the time domain using laguerre polynomials," Computers and Mathematics with Applications, vol. 62, no. 8, pp. 3241-3250, Oct. 2011.

[23] B. Salimbahrami, R. Eid, and B. Lohmann, "Model reduction by second order krylov subspaces: Extensions, stability and proportional damping," 2006 IEEE International Symposium on Intelligent Control, pp. 2997 3002, Oct. 2006.

[24] L. Knockaert, T. Dhaene, F. Ferranti, and D. D. Zutter, "Model order reduction with preservation of passivity, non-expansivity and markov moments." Systems \& Control Letters, vol. 60, no. 1, pp. 53-61, Jan. 2011.

[25] G.Szegö, Orthogonal Polynomials. New York: American Mathematical Society, 1939

[26] W. E. Arnoldi, "The principle of minimized iterations in the solution of the matrix eigenvalue problem," Quarterly of Applied Mathematics, vol. 9, 1951.

[27] E. J. Grimme, Krylov Projection Methods For Model Reduction. Electrical Engineering, University of Illinois: PhD Thesis, 1997.

[28] A. H. Al-mohy and N. J. Higham, "Computing the action of the matrix exponential, with an application to exponential integrators," Electronic Transactions on Numerical Analysis, vol. 37, pp. 147-165, 2010.

[29] N. N. Lebedev, Special functions and their applications. 308 Seiten: Courier Dover Publications, 1972.

[30] K. Zhou, J. C. Doyle, and K. Glover, Robust and Optimal Control. Englewood Cliffs, New Jersey: PrenticeHall, 1996.

[31] A. C. Antoulas, D. C. Sorensen, and Y. Zhou, "On the decay rate of hankel singular values and related issues," CONTROL LETT, vol. 46, pp. 323-342, 2002.

[32] R. W. Freund, "SPRIM structure-preserving reduced-order interconnect macromodeling," Proceedings of the 2004 IEEE/ACM International conference on Computer-aided design, pp. 80-87, 2004.

[33] K. Tsuyoshi and G. Sanjay, Moment Matching Theorems for Dimension Reduction of Higher-Order Dynamical Systems via Higher-Order Krylov Subspaces. University of California, Berkeley: Report No.UCB/SEMM2008/04, 2008.

[34] E. Bogatin, L. Simonovich, S. Gupta, and M. Resso, "Practical analysis of backplane vias," in Proc. DesignCon, pp. 1-24, Feb. 2009.

[35] L. Simonovich, E. Bogatin, and Y. Cao, "Differential via modeling methodology," IEEE Transactions on Components, Packaging and Manufacturing Technology, vol. 1, no. 5, pp. 722-730, May 2011. 


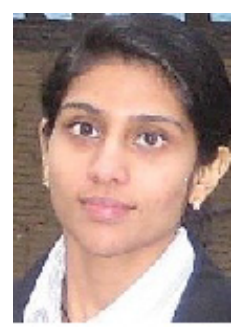

Elizabeth Rita Samuel received the Bachelor of Technology (B.Tech) degree and the Master of Technology (M.Tech) degree in Electrical and Electronics engineering from the University of Kerala, Kerala, India in 2002 and 2005 respectively. She is currently working toward the Ph.D. degree at the Department of Information Technology, Ghent University, Ghent, Belgium. Her current research interests include parametric macromodeling and parameterized model order reduction.

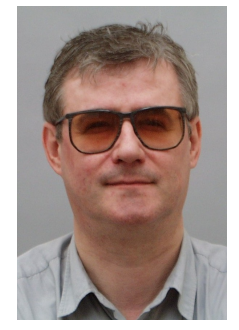

Luc Knockaert (SM'00) received the M. Sc. Degree in physical engineering, the M. Sc. Degree in telecommunications engineering and the $\mathrm{Ph}$. D. Degree in electrical engineering from Ghent University, Belgium, in 1974, 1977 and 1987, respectively. From 1979 to 1984 and from 1988 to 1995 he was working in North-South cooperation and development projects at the Universities of the Democratic Republic of the Congo and Burundi. He is presently affiliated with the Interdisciplinary Institute for Broad Band Technologies (www.ibbt.be) and a professor at the Dept. of Information Technology, Ghent University (www.intec.ugent.be) His current interests are the application of linear algebra and adaptive methods in signal estimation, model order reduction and computational electromagnetics. As author or co-author he has contributed to more than 100 international journal and conference publications. He is a member of MAA, SIAM and a senior member of IEEE.

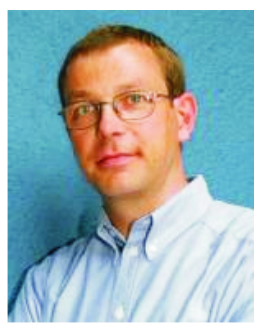

Tom Dhaene (SM'05) was born in Deinze, Belgium, on June 25, 1966. He received the Ph.D. degree in electrotechnical engineering from the University of Ghent, Ghent, Belgium, in 1993. From 1989 to 1993, he was Research Assistant at the University of Ghent, in the Department of Information Technology, where his research focused on different aspects of full-wave electro-magnetic circuit modeling, transient simulation, and time-domain characterization of high-frequency and high-speed interconnections. In 1993, he joined the EDA company Alphabit (now part of Agilent). He was one of the key developers of the planar EM simulator ADS Momentum. Since September 2000, he has been a Professor in the Department of Mathematics and Computer Science at the University of Antwerp, Antwerp, Belgium. Since October 2007, he is a Full Professor in the Department of Information Technology (INTEC) at Ghent University, Ghent, Belgium. As author or co-author, he has contributed to more than 250 peer-reviewed papers and abstracts in international conference proceedings, journals and books about computational science and engineering, numerical analysis, and computer science. He is the holder of 5 U.S. patents. 